\title{
LAMIPPE (LAMIPPE) BOULIGANDI SP. NOV., COPEPODE PARASITE D'OCTOCORALLIAIRE DE LA MER DU LABRADOR ${ }^{1}$ )
}

\section{PAR}

\section{LUCIEN LAUBIER}

Centre Océanologique de Bretagne, B.P. 337, Brest-29 N, France

$\mathrm{Au}$ cours de la campagne Noratlante du navire océanographique Jean Charcot dans l'Atlantique Nord, $j$ 'ai eu l'occasion de récolter dans un Pennatulaire profond une dizaine d'exemplaires d'un Copépode endoparasite appartenant à la famille des Lamippidae de Zulueta, 1908. Au sein de cette famille relativement homogène, dont tous les représentants sont des endoparasites libres ou gallicoles d'Octocoralliaires, la forme rencontrée en Mer du Labrador constitue une espèce nouvelle remarquable par ses caractéristiques morphologiques. La description de cette forme nouvelle, et les remarques systématiques entrainées par sa création, font l'objet du présent travail.

Certaines particularités morphologiques m'avaient tout d'abord conduit à envisager pour cette forme la création d'un genre nouveau et c'est en ce sens qu'avait été préparé le premier manuscrit. Sur l'avis de Y. Bouligand ${ }^{2}$ ), j'ai finalement renoncé à cette manière de voir: en effet, la plupart des caractères pouvant être retenus pour justifier la création d'un genre nouveau concernent des structures articulaires non sclérifiées. Les structures appendiculaires sclérifiées, qui ont une valeur phylétique beaucoup plus forte, ne sont pas génériquement différentes chez le Lamippide de la Mer du Labrador et chez Lamippe (Lamippe) rubra Bruzelius, 1858 par exemple.

D'une manière générale, la famille des Lamippidae est relativement homogène. Les travaux modernes de Bouligand (1960a, 1960b, 1961, 1965, 1966) et de Bouligand \& Delamare Deboutteville (1959a, 1959b), fondés sur l'étude de quinze espèces parmi les vingt-deux espèces valides que compte actuellement la famille des Lamippidae, ont finalement conduit Bouligand (1966) à ne conserver que deux genres, Lamippe Bruzelius, 1858 et Linaresia Zulueta, 1908, les quatre autres genres antérieurement décrits (Isidicola Gravier, 1914, Enalcyonium Olsson, 1868, Lamippella Bouligand \& Delamare Deboutteville, 1959, Lamippina Bouligand, 1960) étant considérés comme des sous-genres de Lamippe, un nouveau sous-

1) Résultats scientifiques de la campagne „Noratlante" du N.O. Jean Charcot en Atlantique Nord, août-septembre-octobre 1969, publication $n^{\circ} 11$.

2) A qui j'exprime à nouveau mes remerciements pour l'intérêt qu'il a bien voulu porter à la critique de ce travail. 
genre étant créé au sein des Lamippe pour renfermer quatre espèces déjà connues (Lamippula Bouligand, 1966). L'auteur ne fournit d'ailleurs pas de justifications morphologiques ou phylétiques de cet essai de rendre plus homogène la systématique de la famille, mais sa grande connaissance du groupe ne permet guère de remettre en cause cette conclusion, du moins pour les espèces bien connues. On peut en effet se poser la question en ce qui concerne Lamippe (Isidicola) antarctica Gravier, 1914, espèce gallicole dont la description est assez imparfaite. Certains caractères tels que la segmentation du corps (qui est reconnaissable chez d'autres espèces par la présence de rangées de papilles cuticulaires, mais n'est jamais très marquée chez les femelles), la structure apparemment très simplifiée de l'antennule et de l'antenne (qui tient peut-être à l'imperfection des observations de Gravier), ont sans doute valeur générique; ces arguments morphologiques sont d'ailleurs renforcés par la localisation de cette forme en Antarctique: à l'exception d'une forme non décrite récoltée par l'expédition de la Siboga au niveau de l'équateur (Versluys, 1902), les autres espèces de Lamippidae ont toutes une répartition boréale.

Au sein du vaste genre Lamippe tel qu'il est défini par Bouligand (1966), l'espèce de la Mer du Labrador doit être rangée dans le sous-genre nominatif, qui ne comprenait jusqu'ici qu'une seule espèce, Lamippe (L.) rubra: ces deux formes sont les seules qui possèdent des maxillipèdes bien développés. D'autres caractéristiques communes témoignent de leur lien phylétique: réduction de l'antennule, similarité des $P_{1}$ et $P_{2}$ par exemple, mais la présence des maxillipèdes justifie à elle seule l'inclusion de la forme nouvelle dans le sous-genre Lamippe.

\section{Lamippe (Lamippe) bouligandi sp. nov. ${ }^{1}$}

Types. - Dix spécimens femelles ont été extraits de l'unique hôte récolté dans le prélèvement décrit ci-dessus. Un seul avait entièrement évaginé sa partie antérieure, les autres étant rétractés jusqu'au niveau de la deuxième paire de pattes thoraciques. L'exemplaire évaginé a été coupé entre les deux paires de pattes thoraciques afin de faciliter l'observation de la région céphalique sous diverses orientations. Cet exemplaire a été déposé comme holotype dans les collections du Muséum National d'Histoire Naturelle, Laboratoire des Crustacés, sous le numéro d'entrée 71-02. Les autres spécimens plus ou moins rétractés sont conservés dans la collection de l'auteur.

Hôte. - Antboptilum grandiflorum (Verrill, 1879) ${ }^{2}$ ).

Localité. - Une dizaine de spécimens femelles ont été extraits d'un unique individu hôte, pêché par $63^{\circ} 10^{\prime} \mathrm{N}$ et $53^{\circ} 40^{\prime} \mathrm{W}$ en Mer du Labrador, sur des fonds

1) L'espèce est amicalement dédiée à M. Yves Bouligand, dont les travaux récents sur les Lamippidae ont apporté un ensemble impressionnant de données morphologiques, anatomiques et biologiques nouvelles.

2) Je remercie le Dr. $\mathrm{H}$. Utinomi, du Laboratoire de Biologie Marine de Seto (Université de Kyoto), qui a bien voulu identifier ce pennatulaire. 
vaseux accidentés (blocs erratiques rocheux transportés par les glaces flottantes), par 1210 mètres de profondeur moyenne. L'engin utilisé était un chalut à panneaux Marinovich (type de chalut commercial utilisé pour la pêche des Penaeidae dans le Golfe du Mexique).

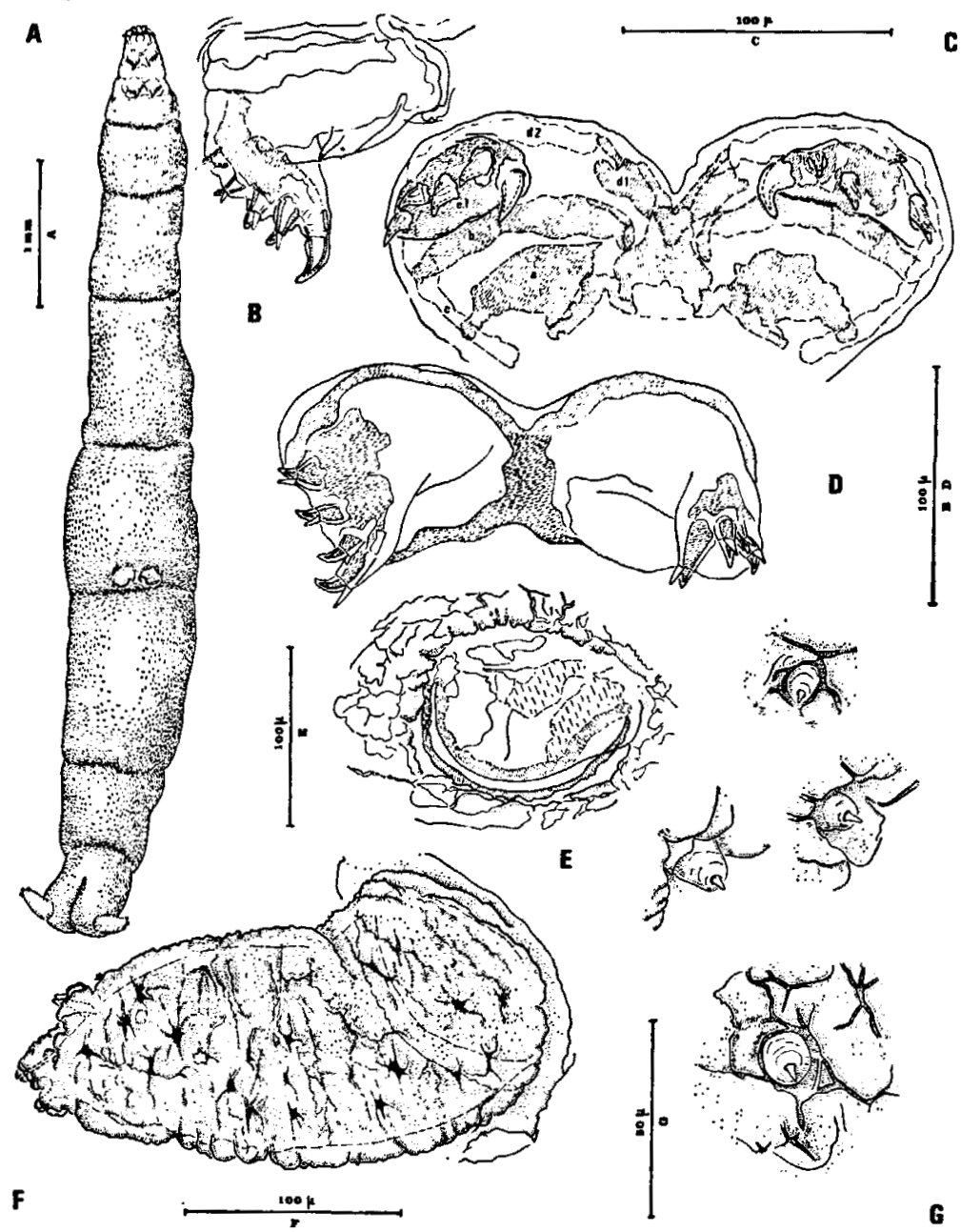

Fig. 1. Lamippe (L.) bouligandi sp. nov. A, habitus en vue ventrale; B, C, P1 en vue antérieure; $D, P_{2}$ en vue antérieure; $E$, vulve génitale droite; $F$, rame furcale droite en vue ventrale; $G$, détail des papilles de la cuticule du corps.

Description. - De grande taille, le corps mesure entre 5 et $7 \mathrm{~mm}$ de longueur selon les individus (fig. 1, A). L'état de retraction ou de sortie de la partie antérieure n'influe sur cette dimension que pour $3 / 10 \mathrm{e}$ de $\mathrm{mm}$ environ, ce qui demeure négligeable en regard des possibilités d'extension ou de contraction de l'ensemble du corps lors de la fixation. Observés sur le vivant lors de la récolte, les animaux sont uniformément blanchâtres; immobiles, ils ne semblent pas doués des remarquables possibilités péristaltiques décrites chez de nombreux Lamippides. Ce fait est à rapprocher de la segmentation marquée du corps. On reconnaît immédiatement les antennules sphériques, les antennes terminées en griffe, le complexe buccal et les deux paires de pattes thoraciques dans la région antérieure. Le premier segment abdominal porte des vulves génitales très postérieures. Enfin, 
le dernier segment abdominal porte les deux rames furcales dirigées transversalement vers l'extérieur. La surface du corps est finement plissée. Il n'y a pas d'acicules

A

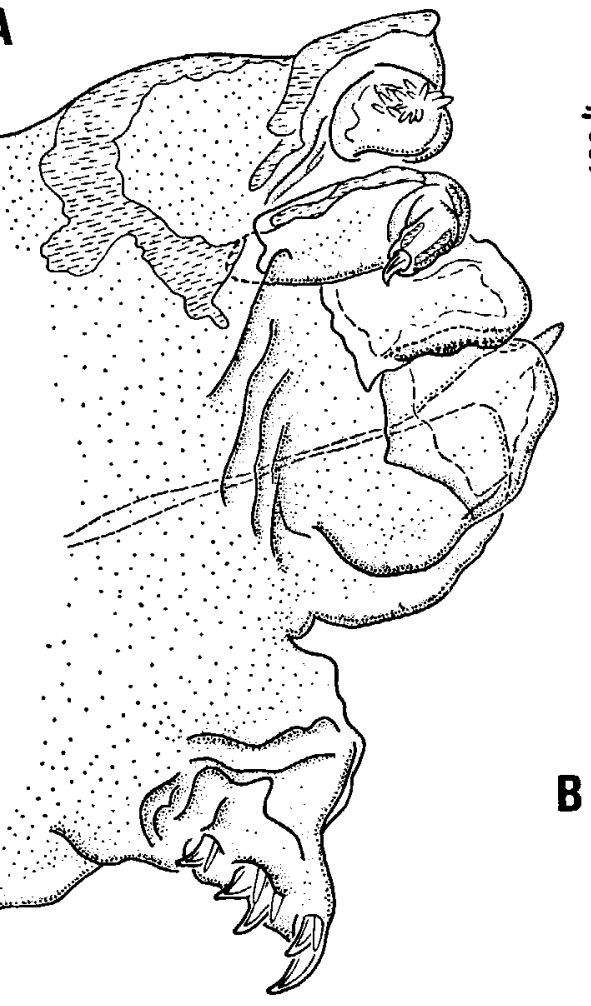

B
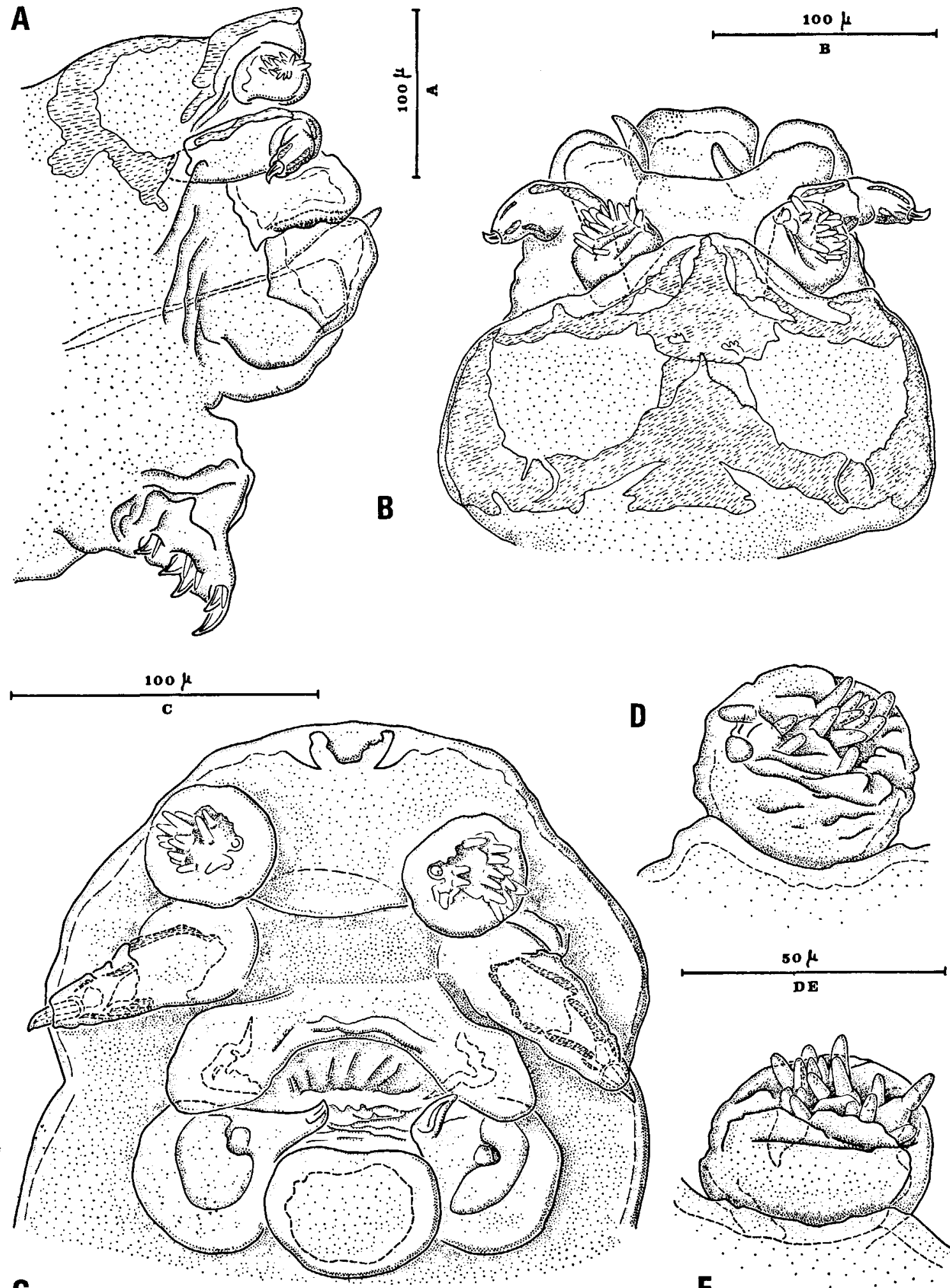

C

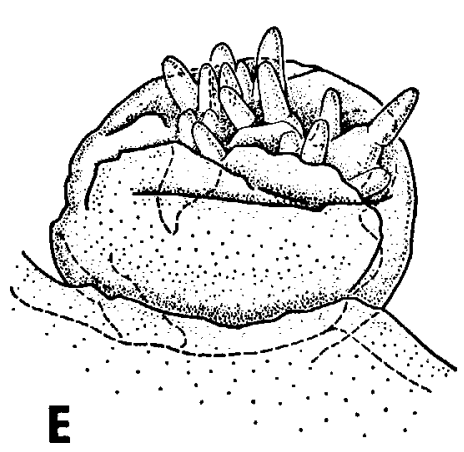

Fig. 2. Lamippe (L.) bouligandi sp. nov. A, B, C, région céphalique en vues latérale, dorsale et apicale; D, antennule droite en vue dorso-apicale; $\mathrm{E}$, antennule gauche en vue dorso-apicale. 


\section{LAMIPPE BOULIGANDI NOV.}

visibles sur les animaux conservés. Ce fait ne permet cependant pas d'affirmer que l'espèce est dépourvue d'acicules. Même en état de décontraction musculaire complète, les acicules de certaines espèces aciculifères demeurent souvent invisibles (Bouligand, in litt.); par ailleurs, $L$. $(L$.$) rubra Bruzelius, seule espèce avec L$. $(L$.) bouligandi $\mathrm{sp}$. $\mathrm{n}$. à posséder des maxillipèdes, porte des acicules (Zulueta, 1908).

Le plan métamérique est donc semblable à celui d'un Lamippe ou de Linaresia (Bouligand, 1966), à l'exception de la région abdominale qui comporte quatre segments bien distincts chez Lamippe $(L$.) bouligandi sp. $\mathrm{n}$. au lieu de trois habituellement.

A
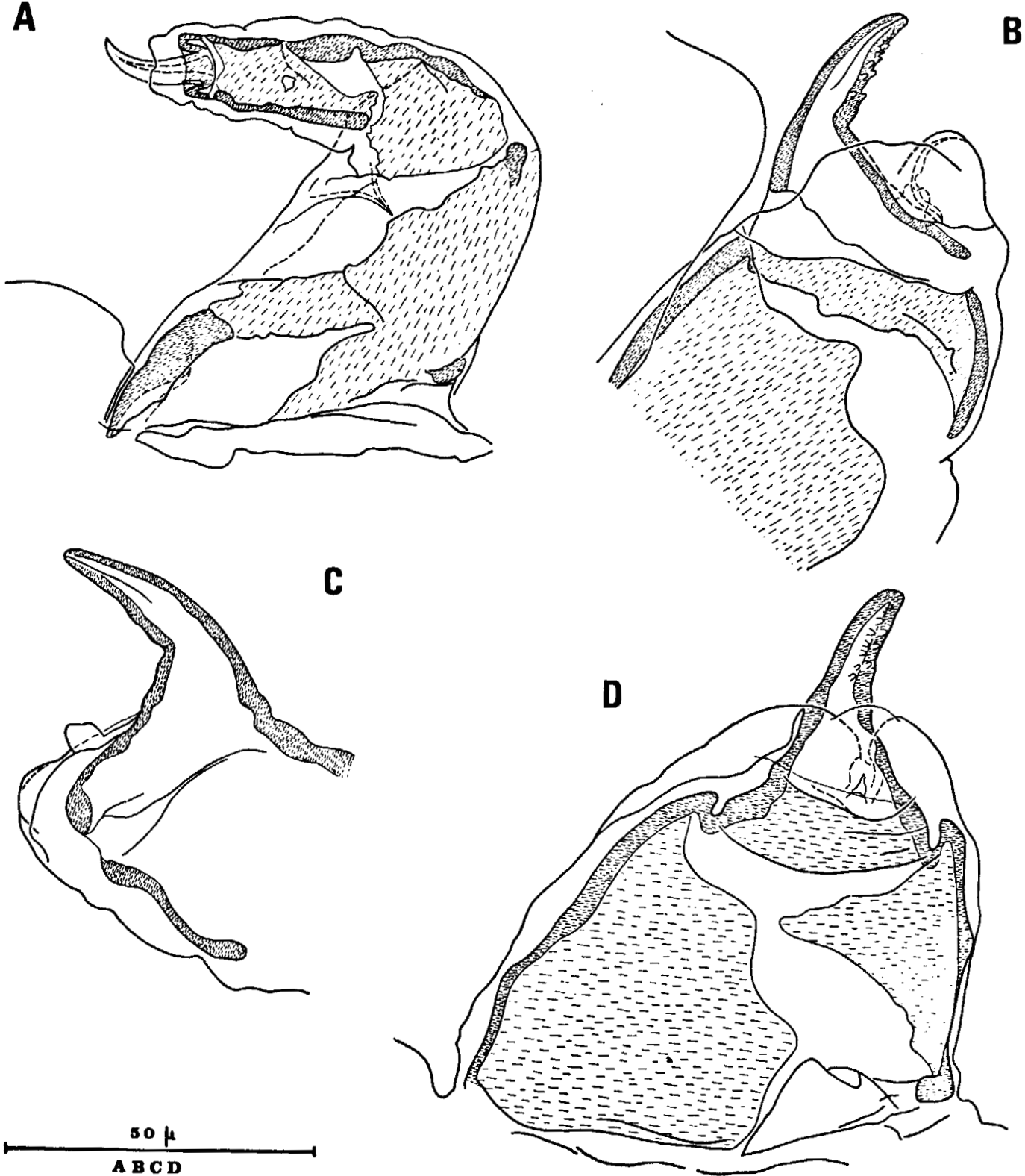

Fig. 3. Lamippe (L.) bouligandi sp. nov. A, antenne gauche en vue antérieure; $B, D$, maxillipède gauche en vues ventrale et latérale; $C$, maxillipède droit en vue ventrale. 
Détail de la morphologie. - Le rostre est bien défini, et fait partie d'un ensemble compliqué de sclérifications nucales. Des deux côtés du rostre sont insérées les antennules (fig. 2, A-E). Ces appendices sont formés d'un unique article globuleux, à sclérifications latérales épaisses, et à calotte distale formée d'une cuticulle mince sans sclérification. Cette zone plus ou moins déprimée et plissíe ${ }^{1}$ ) porte quinze soies cylindro-coniques courtes, à extrémité hémisphérique.

Les antennes sont insérées sous les antennules (fig. 2, A-C, fig. 3, A). Elles sont formées de quatre articles, en considérant la griffe terminale comme un quatrième article. Les trois premiers articles sont définis par trois sclérites distincts, l'articulation la plus importante se situant entre les deux premiers articles. Le troisième article montre à l'extrémité distale une interruption de la sclérification, et il est peut-être plus logique de considérer que l'extrémité distale appartient déjà au quatrième article dont elle constituerait la base. Ces trois articles ne portent ni soies, ni épines. Le dernier article est constitué par la griffe caractéristique des Lamippides, à bord concave lisse. Il est à noter que la griffe antennaire de Lamippe (L.) bouligandi sp. n. ne comporte pas la petite articulation médiane habituellement présente chez les Lamippides et qui a conduit Bouligand à distinguer deux sclérites $\left(4 a_{2}\right.$ et $\left.5 a_{2}\right)$ dans le dernier article antennaire.

Le complexe buccal (fig. 2, A-C) est relativement simple à analyser. Comme Lamippe (L.) rubra Bruzelius, 1858 (Bouligand, 1965), Lamippe (L.) bouligandi $\mathrm{sp}$. n. ne possède entre les deux lèvres antérieure et postérieure qu'une paire d'appendices, les maxillipèdes. La lèvre antérieure forme latéralement deux proéminences dirigées ventralement. Elle est soutenue par des sclérites profonds et difficiles à observer. Le maxillipède biarticulé (fig. 3, B-D), se termine par une griffe puissante portant sur le bord concave externe une série de denticules obtus groupés sur deux génératrices. Latéralement, à la base de cet article distal, on observe un bouton sclérifié masqué par une extension bilobée de l'article proximal. La lèvre postérieure grossièrement cylindrique semble porter à sa face interne des sclérites profonds, et, sous certains angles, deux "paragnathes" dont je n'ai pu vérifier l'existence de façon certaine.

L'ensemble des appendices céphaliques est disposé de haut en bas d'un plan transversal, alors que chez les autres Lamippides, l'orientation oblique correspond davantage au schéma classique des Copépodes.

Les deux paires de pattes thoraciques $P_{1}$ et $P_{2}$ ont une structure classique pour un Lamippide (fig. 1, B-D). Il n'existe pas de rame interne hémisphérique. L'exopodite de $\mathrm{P}_{1}$ porte cinq griffes latérales de taille croissante vers l'extrémité distale. Les quatre griffes proximales sont accompagnées de soies latérales, la grande griffe terminale en est dépourvue. Ce fait intéressant a été vérifié par l'examen sous diverses orientations, et en utilisant le contraste de phase et le contraste interférentiel, sur trois spécimens disséqués. Souvent, les griffes à moitié

1) Le séjour prolongé en acide lactique chaud ne modifie pas l'aspect déprimé de cette zone, il ne s'agit donc probablement pas d'un artéfact lié à la fixation. 
rétractées rendent difficile l'observation, la vue apicale permettant cependant d'acquérir une certitude. L'exopodite de $\mathrm{P}_{2}$ ne porte que quatre griffes disposées de la même manière; les quatre griffes de $\mathrm{P}_{2}$ possèdent des soies latérales accompagnatrices. En vue ventrale, sur $P_{1}$ comme sur $P_{2}$, il est possible de reconnaitre l'ensemble des sclérites décrit par Bouligand (1965) chez Lamippe (L.) rubra: $a, b, c, d_{1}, d_{2}, e, f$ (fig. $1, \mathrm{D}$ ).

Les vulves génitales (fig. 1, E) portent une fente de déhiscence latéro-postérieure de forme classique. Le système de pince interne décrit chez de nombreux Lamippides et dont le rôle est de maintenir le spermatophore n'existe pas chez Lamippe (L.) bouligandi sp. $\mathrm{n}$.

Les branches furcales (fig. 1, F) sont dirigées latéralement des deux côtés du segment anal. De structure assez simple, elles ne portent ni digitations, ni acicules. On distingue seulement à l'extrémité trois formations sclérifiées traversées par un canal axial et terminées en pointe fine, qui peuvent être interprétées comme des digitations aciculifères dont les acicules sont rentrés. Bouligand (1965) a signalé chez Lamippe (L.) rubra, en avant des rames furcales, une formation impaire portant une petite épine antérieure et deux épines plus grandes moyenne et postérieure. Rien de semblable n'existe chez Lamippe (L.) bouligandi sp. n.

Le mâle est inconnu.

Justification. - Il n'est pas possible d'inclure dans le tableau comparatif proposé par Bouligand (1966) Lamippe (L.) bouligandi sp. n., en particulier par suite de la difficulté qu'il y a à reconnaître avec certitude les sclérites appendiculaires des divers appendices. Il est cependant intéressant de souligner les différences principales entre Lamippe (L.) rubra et la nouvelle espèce du sous-genre.

Antennules: en dehors de la forme sphérique de l'antennule de Lamippe ( $L$.) bouligandi sp. n., la distinction repose essentiellement sur le nombre de soies: 15 chez cette dernière, contre 13 chez Lamippe (L.) rubra.

Antennes: assez semblables à l'exception de la pseudo-articulation de la griffe terminale.

Maxillipèdes: semblables.

$P_{1}$ et $P_{2}$ : structure semblable des sclérifications, et même nombre de griffes, mais distribution et nombre des soies latérales différents.

Rames furcales: différence dans le nombre des digitations aciculifères.

Métamérie: deux, peut-être trois segments abdominaux chez Lamippe (L.) rubra contre quatre chez Lamippe $(L$.$) bouligandi sp. n.; d'une façon générale, segmen-$ tation du corps nettement marquée chez cette dernière.

Une étude comparée des deux espèces conduirait probablement à mettre en évidence d'autres caractères distinctifs; ceux qui viennent d'être énumérés justifient amplement la création d'une espèce nouvelle. 
Remarques. - L'espèce qui vient d'être décrite a certainement été déjà observée. En effet, Jungersen (1904) signale la présence dans le même hôte Anthoptilum grandiflorum d'un Copépode parasite: „I flere af Exemplarerne, fra begge Stationerne, fandtes ejendommelige ormelignende Parasiter - til Dels ragende ud af Polypernes Munde; det er snyltende Kopepoder af Slaegten Lamippe Bruz.; øjensynlig en egen Art, som jeg her giver Navnet L. anthoptili" (p. 63). Les hôtes ont été récoltés dans trois stations ( ${ }^{\circ} 6,7$ et 8 de l'Ingolf) au Sud de l'Islande, par 90, 600 et 136 brasses de profondeur, soit pour la station la plus profonde à une profondeur comparable à celle à laquelle a été trouvé $L$. (L.) bouligandi $\mathrm{sp}$. $\mathrm{n}$. Jungersen ne précise pas dans quelles stations ont été observés les parasites, et $n^{\prime}$ accompagne d'aucune description, définition ou indication ${ }^{1}$ ) son observation. A ma connaissance, cette espèce n'a été citée à nouveau que plus de soixante ans après par Bouligand (1966), qui souligne l'absence de toute description ou illustration. Au sens du Code international de Nomenclature zoologique (Arts. 12 et 16), le nom Lamippe antboptili n'est pas utilisable. Cependant, l'observation de Jungersen peut probablement être retenue en ce qui concerne la répartition géographique de $L$. (L.) bouligandi sp. n.

La plupart des Lamippides connus actuellement sont parasites d'Octocoralliaires de faible profondeur. En dehors de Lamippe (L.) bouligandi sp. n., une seule espèce a été récoltée à plus de $1000 \mathrm{~m}$ de profondeur: il s'agit de Lamippe (Enalcyonium) beegaardi Bouligand, 1960, décrit par Heegaard (1949) sous le nom de Lamippe rubra, recueilli dans une Gersemia rubiformis récoltée par l'Ingolf par 2258 mètres de profondeur en Mer du Labrador $\left(63^{\circ} 06^{\prime} \mathrm{N}\right.$ et $\left.56^{\circ} 00^{\prime} \mathrm{W}\right)$. Bouligand (1960: 260 ) signale à propos de cette espèce les caractères qui la distinguent de Lamippe (L.) rubra: la furca de Lamippe (E.) heegaardi est très simplifiée, et les pattes thoraciques présentent beaucoup moins de griffes. On peut ajouter à ces caractères la structure et la chétotaxie de l'antennule par exemple. Ces mêmes arguments morphologiques permettent de séparer facilement Lamippe (L.) bouligandi sp. n. de Lamippe (E.) beegaardi, qui demande d'ailleurs à être redécrite; en particulier, Heegaard (1949) décrit chez cette espèce une paire de mandibules, dont la représentation malheureusement fort schématique rappelle plutôt les maxillipèdes caractéristiques du sous-genre Lamippe (et non du sous-genre Enalcyonium dans lequel l'espèce a été rangée).

D'autres parasites ont parfois été observés dans des Octocoralliaires provenant de grandes expéditions océanographiques, mais dans un état tel qu'ils n'ont pu être décrits (Versluys, 1902; Nutting, 1905; Leigh-Sharpe, 1934 par exemple). Il est probable que le développement des recherches en mer profonde apportera d'autres découvertes du même ordre, mais il est bien évident qu'à l'heure actuelle nos connaissances en ce domaine restent très imparfaites.

1) D'après le Code international de Nomenclature zoologique (1964, Art. 16 (b) (i)), la mention de l'hôte d'un parasite ne constitue pas une ,indication". 


\section{LAMIPPE BOULIGANDI NOV.}

\section{SUMMARY}

- A new species of Lamippidae (Copepoda) has been found in the bathyal pennatulid, Anthoptilum grandiflorum (Verrill, 1879), in the Labrador Sea. The new species is, because of the presence of maxillipeds, attributed to the genus Lamippe, subgenus Lamippe. $L$. (L.) bouligandi sp. nov. is described and a number of taxonomic and zoogeographic remarks are made. -

\section{BIBLIOGRAPHIE}

Bouligand, Y., 1960a. Sur l'organisation des Lamippides. Vie Milieu, 11: 335-380.

- 1960b. Notes sur la famille des Lamippidae, 1. Crustaceana, 1: 258.278.

-, 1961. Notes sur la famille des Lamippidae, 2. Crustaceana, 2: 40-52.

-, 1965. Notes sur la famille des Lamippidae, 3. Crustaceana, 8: 1-24.

- 1966. Recherches récentes sur les Copépodes associés aux Anthozoaires. In: The Cnidaria and their evolution. Symp. zool. Soc. London, 16: 267-306.

Bouligand, Y. \& Cl. Delamare Deboutteville, 1959a. Le dimorphisme sexuel de linaresia mammillifera Zulueta 1908, Copépode parasite de l'Octocoralliaire Muricea chamaeleon von Koch. C. r. hebd. Séanc. Acad. Sci. Paris, 248: 286-288.

—_ \& - 1959b. Lamipella faurei n. g. n.sp., considérations morphologiques sur la famille des Lamippidae, Copépodes parasites des Octocoralliaires. C. r. hebd. Séanc. Acad. Sci. Paris, 249: $1807-1809$.

BRuzeirus, R., 1858. Uber einen in der Pennatula rubra lebenden Schmarotzer. Arch. Naturgesch., 25 (1): 286-290.

GRAvIER, Ch., 1914. Isidicola antarctica, Crustacé parasite de quelques Isidae de l'Antarctique Sudamérican. Deuxième Expédition Antarctique Française (1908-1910). Les Alcyonaires, Appendice: $99-100$.

HzegaArd, P., 1949. Notes on parasitic copepods. Vidensk. Meddr. dansk naturh. Foren. 111: 235-245.

International Trust for ZoOlogical Nomenclature, 1964. Code international de Nomenclature zoologique: i-xx, 1-176. (London).

Jungersen, H. F. E., 1904. Pennatulida. Danske Ingolf-Exped., 5 (1): 1-91, pls. I-III.

Leigh-SharPe, W. H., 1934. The Copepoda of the Siboga Expedition, 2. Commensal and parasitic Copepoda. Siboga-Exped. Monograph, 39 (b): 1-43.

Nurring, C. C., 1905. Some abnormalities of growth produced by parasites on Alcyonaria, Science, 21 : $\mathbf{x x}$.

Olsson, P., 1869. Nova genera parasitantia Copepodorum et Platyelminthium. Lunds. Univ. Arsskr., 6 (2) (7): $1-6$.

VERSLUYS, J., 1902. Voorkomen van parasieten in de polypen van eenige diepzee gorgoniden (Siboga-Exped.). Tijdschr. Ned. dierk. Ver., (2) 7: iii-iv.

ZUIUETA, A. DE, 1908. Note préliminaire sur la famille des Lamippides, Copépodes parasites des Alcynaires. Archs. Zool. exp. gén., (4) 9: 1-30. 